\title{
Performance Analysis of Coherent Interference Suppression Schemes by an Adaptive Antenna Array
}

\author{
Salah Sayed Elagooz ${ }^{*}$
}

\begin{abstract}
Rejection of coherent interference requires an array consisting of several identical sub-arrays which operates with spatial smoothing algorithms. For the cases in which such array configuration cannot be obtained, other schemes must be employed to decouple the desired signal and the coherent interference. This paper presents a coherent interference suppression scheme, known as, the complementally transformed minimum variance beamformer, and compare its behavior with the ordinary multiple constrained minimum variance beamformer. It should be noted that both schemes need prior knowledge of the direction of arrival of the interferences. The behavior of both interference suppression schemes are evaluated through computer simulation. The simulation results indicate the advantage of the complementally transformed minimum variance beamformer over the ordinary multiple constrained minimum variance beamformer from the point of view of the degree of coherent interference reduction.
\end{abstract}

Keywords: Coherent interference suppression scheme - complementally transformed minimum variance beamformer - multiple constrained minimum variance beamformer

\section{I-Introduction:}

Conventional adaptive beamformers achieve high output signal to noise and interference (SINR) as long as the interferers are uncorrelated with the desired signal and the errors in the steering vector (due to pointing or calibration inaccuracy) are small [1,2]. In the presence of steering vector errors and/or correlated interferers, those baemformers exhibit severe degradation in performance. In some extreme cases, such as with a large pointing error or multipath interference, this degradation approach the case of desired signal cancellation $[2,3]$. Trials have been proposed to decrease the effect of desired signal cancellation. For example, to avoid such degradation, the spatial smoothing techniques can be incorporated as a mean of decorrelating the interfering signals before beamforming [3,4]. This ensures that the beamformer effectively nullify all of the interferers. A major restriction of using spatial smoothing techniques is that they require uniform array or an array consisting of several identical subarrays [5,6]. A multiple constrained minimum variance (MCMV) beamformer is proposed, which suppressed coherent interferers by inserting "hard nulls" in their hypothesized direction of arrivals (DOAs). However, this approach is generally sensitive to errors in the DOAs estimates [7]. To reduce this problem, high order constrains can be

\footnotetext{
* Egyptian Armed Forces
} 
incorporated to broaden the effective angular region of operation [6,7]. Unfortunately, increasing the number of constrains results in poorer SINR performance since the effective degree of freedom for suppressing the uncorrelated interference and noise is reduced. The aforementioned problems prompt the development of complementally transformed minimum variance beamformer (CTMV) beamformer [8], which does not require hard nulling for coherent interferers via decoupling the desired signal from the other coherent interferers. The paper is organized as follows: Section II introduces the signal model for analyzing the presented schemes. Section III discusses briefly the analytical study for multiple constrained minimum variance (MCMV) beamformer scheme for nullifying the interference DOA estimation of coherent and non-coherent signals. Section IV discusses the proposed CTMV scheme for extraction of signal of interest (SOI) and nullifying the interference coherent and non-coherent ones. Section V presents the simulations and performance evaluation of the two proposed schemes. Finally, the conclusion is provided in section VI.

\section{II- Signal model}

Suppose that there are a (known/estimated) number of signals $s_{l}(t), \ldots \ldots \ldots \ldots, s_{q}(t)$ all centered on a known frequency, say $f_{c}$, impinging on M-elements antenna array with DOAs $\varphi_{i}$, $i=1,2, \ldots ., q$. These signals may be uncorrelated, as for the signals coming from different signal sources, or can be fully correlated as in multipath propagation, where each path forms a scaled and time-delayed version of the original transmitted signal, or can be partially correlated due to the noise corruption. The intercepted signal by the $i^{\text {th }}$ array element of the array is a superposition of the complex envelope of all the impinging signals, $\widetilde{s}_{k}(t) \forall k=1,2, \ldots \ldots, q$, and an adaptive white Gaussian noise (AWGN) signal, $\widetilde{n}_{i}(t)$,of variance $\sigma_{n}^{2}$. Therefore, the intercepted signal on the $i^{\text {th }}$ element of the linear array, of inter separation between its elements $d$ is given by [9-12]:

$$
x_{i}(t)=\sum_{k=1}^{q} \widetilde{s}_{k}\left(t+\frac{(i-1)}{c} d \sin \phi_{k}\right)+\widetilde{n}_{i}(t) \forall i=1,2, \ldots . ., M
$$

Also, the input data vector for narrowband incident signals may be expressed as [6]:

$$
\mathbf{x}(t)=\sum_{i=1}^{q} \mathbf{a}\left(\phi_{i}\right) \widetilde{s}_{i}(t)+\mathbf{n}(t)
$$

Where, $\mathbf{a}\left(\varphi_{i}\right)$ is the steering vector of the $i^{\text {th }}$ incident signal with DOA, $\varphi_{i}$

$$
\mathbf{a}\left(\phi_{i}\right)=\left[\begin{array}{c}
1 \\
e^{\frac{-j 2 \pi}{\lambda} d \sin \left(\phi_{i}\right)} \\
\cdots \\
e^{\frac{-j 2 \pi}{\lambda}(M-1) d \sin \left(\phi_{i}\right)}
\end{array}\right]
$$

where, $\lambda$ is the signal wave length. In a matrix notation, (2) becomes

$$
\mathbf{x}(t)=\mathbf{A}(\phi) \mathbf{s}(t)+\mathbf{n}(t)
$$

where $\mathbf{A}(\phi)$ is the $M X q$ matrix of the steering vectors 


$$
\mathbf{A}(\phi)=\left[\mathbf{a}\left(\phi_{1}\right), \ldots \ldots \ldots \ldots \ldots \ldots, \mathbf{a}\left(\phi_{q}\right)\right]^{T}
$$

$\mathbf{n}(t)$ is the complex envelope vector of an AWGN signals along the array elements ,

$$
\mathbf{n}(t)=\left[\widetilde{n}_{1}(t), \ldots \ldots \ldots \ldots . ., \tilde{n}_{M}(t)\right]^{T}
$$

and $\mathbf{s}(t)$ is the complex envelope vector of the incident signals

$$
\mathbf{s}(t)=\left[\widetilde{s}_{1}(t), \ldots \ldots \ldots \ldots . ., \widetilde{s}_{q}(t)\right]^{T}
$$

\section{III-Analytical Study of MCMV Nulling Scheme}

This scheme first takes the estimates of the DOAs of the coherent interference signals (from a pre-DOA estimation stage like modified Multiple Signal Classification MUSIC algorithm [9-12]), and then adapts the weights, which are connected to the array elements, by minimizing the array output power subject to null constraints in the directions of the coherent interferences. In this way the coherent interference is suppressed by the null constraints, and the incoherent interference is suppressed by minimizing the array output power. Assuming that there are a desired signal coming from the direction $\varphi_{0}$, and $\mathrm{K}$ coherent interferences and $\mathrm{G}$ incoherent interferences are present at an $\mathrm{M}$ element array, the input vector in (1) can be represented as [13]

$$
\mathrm{x}(\mathrm{t})=\mathrm{a}\left(\phi_{0}\right) \mathrm{s}_{0}(\mathrm{t})+\sum_{\mathrm{k}=1}^{\mathrm{K}} \mathrm{a}\left(\phi_{\mathrm{k}}\right) \rho_{\mathrm{k}} \mathrm{s}_{0}(\mathrm{t})+\sum_{\mathrm{g}=1}^{\mathrm{G}} \mathrm{a}\left(\phi_{\mathrm{g}}\right) \mathrm{s}_{\mathrm{g}}(\mathrm{t})+\mathrm{n}(\mathrm{t})
$$

where

$\mathrm{a}\left(\phi_{\mathrm{k}}\right)$ is the steering vector of the $\mathrm{k}^{\text {th }}$ coherent interference.

$a\left(\phi_{g}\right)$ is the steering vector of the $\mathrm{g}^{\text {th }}$ uncorrelated interference.

$\rho_{\mathrm{k}} \quad$ is the relative amplitude and phase between the $\mathrm{k}^{\text {th }}$ coherent interference and the desired signal.

To suppress coherent and incoherent interference, one generate a weight vector, $W$ that minimizes the array output power, defined as

$$
\mathrm{P}_{\mathrm{r}}=\mathrm{W}^{\mathrm{H}} \mathrm{R}_{\mathrm{K}} \mathrm{W}
$$

Subject to the generalized constraint

$$
\mathrm{C}_{\mathrm{K}} \mathrm{W}=\mathrm{f}_{\mathrm{K}}
$$

where, $\boldsymbol{R}_{\boldsymbol{K}}$ is the covariance matrix defined as $[5,7]$

$$
\begin{aligned}
R_{K}= & E\left[\left|s_{0}(t)\right|^{2}\right]\left(a\left(\phi_{0}\right)+\sum_{k=1}^{K} \rho_{k} a\left(\phi_{k}\right)\right)^{*}\left(a\left(\phi_{0}\right)+\sum_{k=1}^{K} \rho_{k} a\left(\phi_{k}\right)\right)^{\mathrm{T}} \\
& +\sum_{g=1}^{G} E\left[\left|s_{g}(t)\right|^{2}\right] a^{*}\left(\phi_{g}\right) a^{\mathrm{T}}\left(\phi_{g}\right)+\sigma^{2} I
\end{aligned}
$$

$C_{K}$ is a ${ }^{M \times(K+1)}$ matrix which is defined as 


$$
\mathrm{C}_{\mathrm{K}}=\left[\begin{array}{lllll}
\mathrm{a}\left(\phi_{0}\right) & \mathrm{a}\left(\phi_{\mathrm{c} 1}\right) & \mathrm{a}\left(\phi_{\mathrm{c} 2}\right) & \ldots & \mathrm{a}\left(\phi_{\mathrm{cK}}\right)
\end{array}\right]
$$

the set of constraints $\mathrm{f}_{\mathrm{K}}$ is $a(K+1) \times 1$ vector, and it is defined as

$$
f_{K}=\left[\begin{array}{llllll}
1 & 0 & 0 & 0 & \ldots \ldots \ldots \ldots . . . .0
\end{array}\right]^{T}
$$

The weight vector that achieves (10) can be obtained using various algorithms which give the solution as $[5,7]$

$$
\mathrm{W}_{\mathrm{K}}=\mathrm{R}_{\mathrm{K}}^{-1} \mathrm{C}_{\mathrm{K}}\left(\mathrm{C}_{\mathrm{K}}^{\mathrm{H}} \mathrm{R}_{\mathrm{K}}^{-1} \mathrm{C}_{\mathrm{K}}\right)^{-1} \mathrm{f}_{\mathrm{K}}
$$

\section{IV- Analytical Study of CTMV Nulling Algorithm}

This scheme does not require hard nulls for the coherent interference. To work without hard nulling constraints it is necessary to decouple the desired signal from other coherent interferes in order to avoid mutual cancellation. To achieve this, the CTMV adaptive array processor performs the following steps:

At first, employs a transformation $\mathrm{T}$ to remove the desired signal and retain the coherent interference using DOAs estimates. This transformation is constructed so as to minimize the difference between the original and transformed data subject to the aforementioned complement constraints.

The transformed data (which contains only interference and noise) is then sent to a regular minimum variance distortionless response beamformer (MVDR) which computes the weight vector yielding the maximum output SINR[2,6,9,12], thus the beamformer will perform a mutual cancellation for the coherent interferences solely (because the desired signal is removed before this step). This is in contrast to the regular (MVDR) which work without removing the desired signal at first and so it performs a mutual cancellation between the desired signal and the coherent interference. To decouple the desired signal from the coherent interference signals we use an $M \times M$ linear transformation T satisfying that

$$
\begin{array}{ll}
\operatorname{Ta}\left(\phi_{0}\right)=0_{M} & \\
\operatorname{Ta}\left(\phi_{i}\right)=a\left(\phi_{i}\right) & i=1, \ldots . K \\
\operatorname{Ta}\left(\phi_{i}\right)=\operatorname{Ta}\left(\phi_{i}\right) & i=K+1, \ldots . G+K
\end{array}
$$

Such that

$$
T x=0+\sum_{i=1}^{K} s_{i} a\left(\phi_{i}\right)+\sum_{i=K+1}^{G+K} s_{i} T a\left(\phi_{i}\right)+T n
$$

$\boldsymbol{T}$ is a complementally transformation (CT) matrix.

In order that the beamformer works properly, we must minimize the error between the original signal and the transformed one. This error is given by $[8,12]$

$$
\varepsilon=\mathrm{E}\left\{\|\mathrm{Tx}-\mathrm{x}\|^{2}\right\} \equiv \operatorname{tr}\left\{(\mathrm{T}-\mathrm{I}) \mathrm{R}_{\mathrm{x}}(\mathrm{T}-\mathrm{I})^{\mathrm{H}}\right\}
$$

where, $\|$.$\| and \operatorname{tr}\{$.$\} denote the vector norm and the trace operator respectively.$ 
Incorporation of the linear constraints of (15) in the minimization of (17) with replaced $\varphi_{i}, s$ by their estimates $\hat{\varphi}_{i}, s$ leads to the following constrained problem $[6,8,12]$ $\min _{\mathrm{T}} \operatorname{tr}\left\{(\mathrm{T}-\mathrm{I}) \mathrm{R}_{\mathrm{x}}(\mathrm{T}-\mathrm{I})^{\mathrm{H}}\right\}$ subject to : $\mathrm{T} \hat{\mathrm{A}}_{\mathrm{C}}=\hat{\mathrm{B}}_{\mathrm{C}}$

where

$$
\begin{gathered}
\hat{A}_{C}=\left[a\left(\hat{\phi}_{0}\right), a\left(\hat{\phi}_{1}\right), \cdots, a\left(\hat{\phi}_{K}\right)\right] \\
\hat{B}_{C}=\left[0_{M}, a\left(\hat{\phi}_{1}\right), \cdots, a\left(\hat{\phi}_{K}\right)\right]
\end{gathered}
$$

Thus, the optimum beamforming weight vector is determined via the MVDR criterion acting on the complementally transformed data Tx. Thus this optimum weight vector will be the solution of the following problem[6,8,12]:

$$
\begin{aligned}
& \min _{\text {w }} \mathrm{E}\left\{\left|\mathrm{W}^{\mathrm{H}} \mathrm{Tx}\right|^{2}\right\} \equiv \mathrm{W}^{\mathrm{H}} \mathrm{TR}_{\mathrm{x}} \mathrm{T}^{\mathrm{H}} \mathrm{W} \\
& \text { subject to } \mathrm{W}^{\mathrm{H}} \mathrm{A}\left(\hat{\phi}_{0}\right)=1
\end{aligned}
$$

The optimum weight vector that satisfies the above equation is $[6,8,12]$

$$
\mathrm{W}=\frac{1}{\mathrm{a}^{\mathrm{H}}\left(\hat{\phi}_{0}\right)\left[\mathrm{TR}_{\mathrm{x}} \mathrm{T}^{\mathrm{H}}\right]^{-1} \mathrm{H} \mathrm{a}\left(\hat{\phi}_{0}\right)}\left[\mathrm{TR}{ }_{\mathrm{x}} \mathrm{T}^{\mathrm{H}}\right]^{-1} \mathrm{a}\left(\hat{\phi}_{0}\right)
$$

There are two problems here

$\mathrm{T}$ is not a full rank. Thus, $\mathrm{TR}{ }_{\mathrm{x}} \mathrm{T}^{\mathrm{H}}$ is singular.

The noise component in $\mathrm{TR}_{\mathrm{x}} \mathrm{T}^{\mathrm{H}}$ (which is $\sigma_{\mathrm{n}}^{2} \mathrm{TT}^{\mathrm{H}}$ ) is no longer the same as in the original $\mathrm{R}_{\mathrm{x}}$ (which is $\sigma_{\mathrm{n}}^{2} \mathrm{I}$ ). This suggests that the $\mathrm{CT}$ correlation matrix $\mathrm{TR}_{\mathrm{x}} \mathrm{T}^{\mathrm{H}}$ be modified by a full rank matrix by replacing its noise part with $\sigma_{\mathrm{n}}^{2} \mathrm{I}[6,8,12]$

$$
\tilde{\mathrm{R}}_{\mathrm{x}}=\mathrm{TR}_{\mathrm{x}} \mathrm{T}^{\mathrm{H}}-\sigma_{\mathrm{n}}^{2} \mathrm{TT}^{\mathrm{H}}+\sigma_{\mathrm{n}}^{2} \mathrm{I}
$$

Replacing $\mathrm{TR}_{\mathrm{x}} \mathrm{T}^{\mathrm{H}}$ by $\stackrel{\approx}{\mathrm{R}} \mathrm{x}$ in (22) we obtain

$$
\mathrm{W}=\frac{1}{\mathrm{a}^{\mathrm{H}}\left(\hat{\phi}_{0}\right) \tilde{\mathrm{R}}_{\mathrm{x}}^{-\mathrm{H}^{\mathrm{H}}} \mathrm{a}\left(\hat{\phi}_{0}\right)} \tilde{\tilde{\mathrm{R}}}_{\mathrm{x}}^{-1} \mathrm{a}\left(\hat{\phi}_{0}\right)
$$

\section{V- Simulation Results}

Computer simulations were carried out to evaluate the performance of the CTMV and MCMV beamformers. Several case studies and behavior analysis are presented through the simulations. The following subsections show the simulation results in details. 


\section{A) Case Studies of MCMV Beamformer}

A simulation program has been carried out based on a linear array of eleven elements with

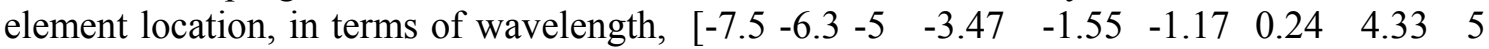
6.5 7.3]. The array is illuminated by the desired signal, a coherent interference, and an incoherent interference with arrival angles $\left[\begin{array}{lll}0^{\circ} & -25^{\circ} & 35^{\circ}\end{array}\right]$ respectively. The three sources are assumed to have equal power which is $10 \mathrm{~dB}$ relative to the background white Gaussian noise. 128 snapshots are taken. The Known /estimated DOAs are then applied to the MCMV beamformer in order to remove the interference. The results presented in Fig. 1 shows a deep null in the direction of the coherent interference and another smaller null in the direction of the uncorrelated interference. This difference in the nulls levels is because that the nulling of the uncorrelated interference comes from minimizing the output power not from the null constraints applied on the coherent interference directions.

\section{B ) Case Studies of CTMV Beamformer}

A computer simulation has been carried to ascertain the performance of the CTMV beamformer. The array employed was a 11-element nonuniform linear array with the following inter element spacing(in terms of wave length) $\left\{0.19,0.19 \times 1.1,0.19 \times 1.1^{2}, \cdots 0.19 \times 1.1^{10}\right\}$. All elements were assumed identical and omni directional with a unit gain. The array is illuminated by the desired signal, a coherent interference, and an incoherent interference with arrival angles $\left[\begin{array}{lll}0^{\circ} & -25^{\circ} & 35^{\circ}\end{array}\right]$ respectively. The three sources are assumed to have equal power which is $10 \mathrm{~dB}$ relative to the background white Gaussian noise. 128 snapshots are taken. The Known / estimated DOAs are then applied to the CTMV beamformer in order to remove the interference. The results presented in Fig. 2 shows two nulls in the directions of the coherent interference and the uncorrelated interference.

\section{C ) Behavior analysis of MCMV and CTMV beamformers}

The performance of the CTMV and MCMV beamformers is evaluated based on the following parameters:

- Input signal to noise ratio $\left(\mathrm{SNR}_{\mathrm{i}}\right)$. - Input Signal to interference ratio $\left(\mathrm{SIR}_{\mathrm{i}}\right)$.

- Number of array elements (M). - Number of snapshots (N).

- DOA estimation error.

The performance measures considered in the simulations are:

- Accuracy of the null (in degree).

- Improvement factor (defined as the ratio between $\mathrm{SIR}_{\mathrm{o}}$ and $\mathrm{SIR}_{\mathrm{i}}$ )

$$
\mathrm{IMF}=\frac{\mathrm{SIR}_{\mathrm{o}}}{\mathrm{SIR}_{\mathrm{i}}}
$$

- Maximum number of cancelled interference sources, (RS). 
Paper: ASAT-13-CM-42

\section{1) The Effect of the Input Signal to Noise Ratio $\left(S_{N R}\right)$}

The effect of increasing the desired signal power on the behavior of the MCMV and CTMV beam formers is considered. It is assumed that there are one desired signal and one coherent interference source at the input of the array. This is for $\mathrm{SIR}_{\mathrm{i}}=0 \mathrm{~dB}, \mathrm{M}=20, \mathrm{~N}=512$, DOA estimation error=0. Table. 1 shows the effect of $\mathrm{SNR}_{\mathrm{i}}$ on both the nulling accuracy and the IMF for both MCMV and CTMV beam formers. From the table we find that the MCMV accuracy is constant at $0.6^{\circ}$ (optimum value), and as $\mathrm{SNR}_{\mathrm{i}}$ increases the IMF decreases. This result occurs because that as $\mathrm{SIR}_{\mathrm{i}}=0$, so if $\mathrm{SNR}_{\mathrm{i}}$ increased then input interference to noise ratio $\left(\mathrm{INR}_{\mathrm{i}}\right)$ would increase which would cause improvement on the performance. This improvement of the performance does not cause better accuracy (table), but makes the null narrower, so we get a narrower null at the same inaccurate angle which will cause worse IMF. Also, the effect of increasing the desired signal power on CTMV behavior is considered. From the table, one find that for $\left(\mathrm{SNR}_{\mathrm{i}}=-10\right.$ to 25$)$ the increasing of $\mathrm{SNR}_{\mathrm{i}}$ will improve the system performance. This is because that $\mathrm{SIR}_{\mathrm{i}}=0$, so increasing $\mathrm{SNR}_{\mathrm{i}}$ means increasing the power of the interference, which would improve the performance. On the other hand, for $\left(\mathrm{SNR}_{\mathrm{i}}=-25\right.$ to-10) one find that, though decreasing $\mathrm{SNR}_{\mathrm{i}}$ means decreasing the interference power, the performance improved. This is because that decreasing $\mathrm{SNR}_{\mathrm{i}}$ means decreasing the power of the desired signal which will make $\mathrm{T}$ very close to I and this will improve the performance and substitut the bad effect of decreasing the interference power.

Moreover the simulations for MCMV performance show that, by increasing $\mathrm{SNR}_{\mathrm{i}}$ the maximum number of cancelled interference increases. That for $\mathrm{SNR}_{\mathrm{i}}=-5$ this number was $R S=10$, and for $\mathrm{SNR}_{\mathrm{i}}=20$ this number was $\mathrm{RS}=11$. This is because as $\mathrm{SIR}_{\mathrm{i}}=0 \mathrm{~dB}$ so increasing $\mathrm{SNR}_{\mathrm{i}}$ means increasing $\mathrm{INR}_{\mathrm{i}}$ and the performance will improve. However, the simulations for CTMV performance show that, by increasing $\mathrm{SNR}_{\mathrm{i}}$ the maximum number of cancelled interference decreases, that for $\mathrm{SNR}_{\mathrm{i}}=-5$ this number was $\mathrm{RS}=3$, and for $\mathrm{SNR}_{\mathrm{i}}=20$ this number was $\mathrm{RS}=2$. This result occurs because increasing the desired signal power will make T no longer approximates I, which will make the system performance worse.

\section{2) The effect of the Input Signal to Interference Ratio (SIRi)}

Table 2 and Table 3 explain the effect of changing the level of the input interference signal, or equivalently the $\operatorname{SIR}_{i}$, on the accuraccy of the null and the IMF. It is assumed that there are one desired signal and one coherent interference at the input of the array. This is for $M=20$, $\mathrm{N}=512$, DOA estimation error $=0$, while the $\mathrm{SNR}_{\mathrm{i}}=-5 \mathrm{~dB}$ and $20 \mathrm{~dB}$ for Table. 3 and Table. 4 respectively. For MCMV performance, one find that increasing $\operatorname{SIR}_{i}$ does not affect the accuracy of the null (because it is already at the optimum value), meanwhile the IMF increases with the increasing of the $\mathrm{SIR}_{\mathrm{i}}$ in the first case $\left(\mathrm{SNR}_{\mathrm{i}}=-5 \mathrm{~dB}\right)$, while it remains constant in the second case $\left(\mathrm{SNR}_{\mathrm{i}}=20 \mathrm{~dB}\right)$. These results can be explained as follwes: As $\mathrm{SIR}_{\mathrm{i}}$ increases, so the interference power relatively decreases and the performance will get worse so the null will get wider but the accuracy remain constant. So we get a wider null at the same inaccurate angle which will allow this null to cover the true angle of the interference, and the IMF will get better.

For CTMV performance, we find that as $\mathrm{SIR}_{\mathrm{i}}$ increases the accuracy and the IMF will get worse. This result occurs because that as $\mathrm{SIR}_{\mathrm{i}}$ increases the interference power decreases, and the null in the direction of the interference will not be suffecient. and then the performance will get worse.

Moreover the simulations show that, by increasing $\mathrm{SIR}_{\mathrm{i}}$ the maximum number of cancelled interference using MCMV beamformer decreases, that for $\mathrm{SIR}_{\mathrm{i}}=-5$ this number was $\mathrm{RS}=12$, and for $\mathrm{SIR}_{\mathrm{i}}=20$ this number was $\mathrm{RS}=11$. However, the maximum number of cancelled 
interference using CTMV beamformer decreases, that for $\mathrm{SIR}_{\mathrm{i}}=-5 \mathrm{~dB}$ this number was $\mathrm{RS}=4$, and for $\mathrm{SIR}_{\mathrm{i}}=20 \mathrm{~dB}$ this number was $\mathrm{RS}=2$.

\section{3) The effect of number of array elements (M)}

Table 4 explains the effect of the number of array elements (M) on the beamformer accuracy and IMF. In this table we suppose that there are one desired signal and one coherent interference signal at the input of the array. This is for $\mathrm{SNR}_{\mathrm{i}}=-5 \mathrm{~dB}, \mathrm{SIR}_{\mathrm{i}}=0 \mathrm{~dB}, \mathrm{~N}=512, \mathrm{DOA}$ estimation error $=0$. The same study is repeated at $\mathrm{SNR}_{\mathrm{i}}=20 \mathrm{~dB}$ without changing the other parameters, the results are listed at Table 5.

From the tables one find that MCMV accuracy is constant at $0.6^{\circ}$, and in the case of $\mathrm{SNR}_{\mathrm{i}}=20$, the IMF decreases as $\mathrm{M}$ increases. This result occurs because the improvement of the performance, caused by increasing $\mathrm{M}$, does not causes better accuracy but makes the null narrower. However, from the two tables, we find that increasing $\mathrm{M}$ improves both accuracy and IMF of CTMV beamformer.

Moreover, the simulations show that, by increasing the number of elements the maximum number of cancelled interference increases, that for MCMV beamformer, for $\mathrm{M}=3$ this number was $\mathrm{RS}=2$, and for $\mathrm{M}=30$ this number was $\mathrm{RS}=14$. Also, for $\mathrm{CTMV}$ beamformer, for $\mathrm{M}=5$ this number was $\mathrm{RS}=1$, and for $\mathrm{M}=30$ this number was $\mathrm{RS}=3$.

\section{4) The Effect of Number of Snapshots (N)}

Table 6 explains the effect of the number of snapshots $(\mathrm{N})$ on the beamformer accuracy and IMF. In this table one suppose that there are one desired signal and one coherent interference source at the input of the array. This is for $\mathrm{SNR}_{\mathrm{i}}=-5 \mathrm{~dB}, \mathrm{SIR}_{\mathrm{i}}=0 \mathrm{~dB}, \mathrm{M}=20, \mathrm{DOA}$ estimation

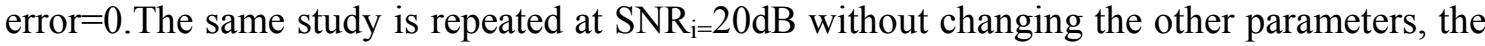
results are explained at Table.7. From the two tables we find that the MCMV beamformer accuracy is constant at $0.6^{\circ}$, and in the case of $\mathrm{SNR}_{\mathrm{i}}=20$ the IMF slightly decreases as $\mathrm{N}$ increases. This is because increasing $\mathrm{N}$ would cause improvement on the performance. But this improvement of the performance does not cause better accuracy but makes the null narrower, so we get a narrower null at the same inaccurate angle. However, for CTMV beamformer, we find that increasing $\mathrm{N}$ improves both accuracy and IMF.

Moreover, the simulations show that, by increasing the number of snapshots the maximum number of cancelled interference increases, that for MCMV beamformer, for $\mathrm{N}=8$ this number was $\mathrm{RS}=6$, and for $\mathrm{N}=512$ this number was $\mathrm{RS}=11$. Also, for $\mathrm{CTMV}$ beamformer, for $\mathrm{N}=32$ this number was $\mathrm{RS}=1$, and for $\mathrm{N}=512$ this number was $\mathrm{RS}=2$.

\section{5) The Effect of DOA Estimation Errors}

Table 8 explains the effect of DOA estimation errors on the beamformer accuracy and IMF. In this table one suppose that there are one desired signal and one coherent interference signal at the input of the array. This is for $\mathrm{SNR}_{\mathrm{i}}=-5 \mathrm{~dB}, \mathrm{SIR}_{\mathrm{i}}=0 \mathrm{~dB}, \mathrm{M}=20, \mathrm{~N}=512$. In the table, the DOA estimation errors are represented in a vector of two elements, the first element is the DOA estimation error of the desired signal and the second element is the DOA estimation error of the coherent interference signal. The same study is repeated at $\mathrm{SNR}_{\mathrm{i}}=20 \mathrm{~dB}$ without changing other parameters. The results are listed at Table.9. From the tables one find that as the DOA estimation error increases both the MCMV accuracy and the improvement factor will get worse, and it is worth to notice that the DOA estimation error of the interference signal is more effective than the DOA estimation error of the desired signal. However, both the CTMV accuracy and the improvement factor will get worse with the same degree. 


\section{6) Wide Band Interference Nulling}

In this section one will investigate the case of presence of wideband interference. The estimated DOAs are then applied to the nulling beamformer (MCMV or CTMV) in order to cancel the interference. A computer simulation has been carried out to investigate the capability of MCMV and CTMV beamformers to remove wideband interference. During the simulation we assume that there are three wideband signals impinging a nonuniform array of fifteen elements.

The inter-element spacing is ( in terms of wavelength) given by

$\left\{0.19,0.19 \times 1.1,0.19 \times 1.1^{2}, \cdots 0.19 \times 1.1^{14}\right\}$

All elements were assumed identical and omni-directional with a unit gain. The first signal is assumed as the desired one and its arrival angle is $0^{\circ}$, the other two signals are assumed as the interference ones, and their arrival angles are $-25^{\circ}$ and $35^{\circ}$. The three signals are assumed to have equal power which is $20 \mathrm{~dB}$ relative to the background noise. 32 snapshots are taken, and the bandwidth of the signals is assumed as the third of the carrier frequency $(\mathrm{B}=0.3 * \mathrm{fr})[6,9]$. After applying estimated DOAs are applied to the studied nulling beamformers, the results are depicted in Fig.(3) for MCMV beamformer and Fig. 4 for CTMV beamformer. Both figures are plotted at the center frequency of the considered bandwidth. From the figures we notice that both MCMV and CTMV beamformers success in steering nulls in the directions of the interference sources. Another case study is considered. In this case one assume the same assumptions of the previous case except that the desired signal considered a narrow band signal. Fig. 5 for MCMV beamformer and Fig. 6 for CTMV beamformer show that both MCMV and CTMV beamformers success steering nulls in the directions of the interference sources. (Both figures are plotted at the center frequency of the considered bandwidth). Finally it is worth to mention that the system accuracy gets worth at the frequencies located at the edges of the considered bandwidth. So it is proposed to connect a taped delay line with each sensor and perform a spatial temporal processing.

\section{VI-Conclusions}

The analytical studies of both the MCMV and the CTMV beamformers have been presented and their behaviors have been evaluated through computer simulations. The simulations results indicate the advantage of the CTMV beamformer over the ordinary MCMV one from the point of view of coherent interference reduction. A comparison between the studied beamformers is summarized as follows:

- Both methods work with uniform and nonuniform arrays.

- Both methods can cancel both uncorrelated and coherent interference.

- Both methods can cancel both narrowband and wideband interference.

- The MCMV beamformer is more accurate than the CTMV beamformer.

- The CTMV beamformer is more robust to the DOA estimation errors than the MCMV beamformer. 


\section{VIII-References}

[1] V. Viikari, V. Kolmonen, J. Salo, Antti V. Raisanen, "Antenna Pattern Correction Technique Based on an Adaptive Array Algorithm”, IEEE trans on antennan and propag, vol. 55, NO. 8, August 2007.

[2] Widrow, K.M Duvall, R.P Gooch, and W.C. Newman.” Signal cancellation phenomena in adaptive antennas: Causes and cures." IEEE Trans. on Antennas and Propagation, Vol. $\mathrm{AP}=30$, pp.469-478, May 1982.

[3] T.J. Shan, M. Wax, T. Kailath, "On Spatial Smoothing for Estimation of Coherent Signals," ICASSP, Vol. ASSP-33, August 1985.

[4] F. Haber, M. Zoltowski, "Spatial Spectrum Estimation in a Coherent Signal Environment Using an Array in Motion," IEEE Trans. on Antennas and Propagation, Vol. AP-34, March 1986, pp. 301-310.

[5] C.C. Yeh and W.D.Waang. , "Coherent interference suppression by an antenna array of arbitrary geometry.” IEEE Trans. Antennas Propagation, Vol.37, pp.1317-1322, Oct. 1989.

[6] D. a Filho, C. C. Cavalcante, L. S. Resende ,T. Romano "On Adaptive LCMV Beamforming for Multiuser Processing in Wireless Systems" IEEE International Microwave \& Optoelectronics Conference 2007.

[7] M. Wax and Y. Anu," Performance analysis of the minimum variance beam former," IEEE Trans. Signal Processing, Vol. 44, pp928-937, April 1996.

[8] Ta .Sung Lee," Coherent Interference Suppression With complementally Transformed Adaptive Beamformer “, IEEE trans. Antennas and Propagation, Vol.46, No.5, pp.609617, May 1998.

[9] H. Krim, Mats Viberg," Two decades of array signal Processing research", IEEE Signal Processing Magazine, pp.67-94, July 1996.

[10] Brown, A. and N. Gerein "Test Results of a Digital Beamforming GPS Receiver in a Jamming Environment," in Proceedings of ION GPS, September, Salt Lake City.

[11] B. K. Lau, M. Viberg, and Y. H. Leung, "Data-Adaptive Array Interpolation for DOA Estimation in Correlated Signal Environments," in Proc. IEEE International Conference on Acoustics, Speech and Signal Processing (ICASSP'05), vol. 4, pp. 945-948, 2005.

[12] Allen, B. and M. Ghavami Adaptive Array Systems, John Wiley \& Sons

[13] J. Xin, A. Sano, " Direction Estimation of Coherent Signals Using Spatial Signature ”, IEEE signal proc, vol. 9, NO. 12, December 2002.

[14] S. Naidu, Prabhaker, "Sensor Array Signal Processing “ CRC Press, 2001.

[15] Andrew Knigh, "Basics of matlab and beyond", Champion Hall, Florida, 2000.

Table (1) The effect of the SNRi on MCMV and CTMV performances $(\mathrm{SIRi}=\mathbf{0 d B})$

\begin{tabular}{|c|c|c|c|c|c|c|c|c|c|c|c|c|}
\hline \multicolumn{2}{|c|}{ SNRi(dB) } & -25 & -20 & -15 & -10 & -5 & 0 & 5 & 10 & 15 & 20 & 25 \\
\hline \multirow{2}{*}{$\sum_{\sum}^{Z}$} & Accuracy & $0.6^{\circ}$ & $0.6^{\circ}$ & $0.6^{\circ}$ & $0.6^{\circ}$ & $0.6^{\circ}$ & $0.6^{\circ}$ & $0.6^{\circ}$ & $0.6^{\circ}$ & $0.6^{\circ}$ & $0.6^{\circ}$ & $0.6^{\circ}$ \\
\hline & $\operatorname{IMF}(\mathrm{dB})$ & 135 & 135 & 135 & 135 & 130 & 125 & 123 & 118 & 116 & 115 & 115 \\
\hline \multirow{2}{*}{$\sum_{E}$} & Accuracy & $1.5^{\circ}$ & $1.5^{\circ}$ & $3.5^{\circ}$ & $4.5^{\circ}$ & $3.5^{\circ}$ & $2.5^{\circ}$ & $1.5^{\circ}$ & $1^{\circ}$ & $0.75^{\circ}$ & $0.6^{\circ}$ & $0.6^{\circ}$ \\
\hline & $\operatorname{IMF}(\mathrm{dB})$ & 22.5 & 20 & 17 & 16 & 17 & 20 & 22 & 24 & 29 & 32 & 35 \\
\hline
\end{tabular}


Table (2) The effect of the SIRi on MCMV and CTMV performances

$(\mathrm{SNRi}=-\mathbf{5 d B})$

\begin{tabular}{|l||l||l|l|l|l|l|l|l|l|l|l|l|}
\hline \hline \multicolumn{2}{|l|}{$\operatorname{SIRi}(\mathrm{dB})$} & -25 & -20 & -15 & -10 & -5 & 0 & 5 & 10 & 15 & 20 & 25 \\
\hline \hline \multirow{2}{*}{$\sum_{\Sigma}^{Z}$} & Accuracy & $0.6^{\circ}$ & $0.6^{\circ}$ & $0.6^{\circ}$ & $0.6^{\circ}$ & $0.6^{\circ}$ & $0.6^{\circ}$ & $0.6^{\circ}$ & $0.6^{\circ}$ & $0.6^{\circ}$ & $0.6^{\circ}$ & $0.6^{\circ}$ \\
\cline { 2 - 12 } & $\operatorname{IMF}(\mathrm{dB})$ & 125 & 125 & 126 & 128 & 130 & 130 & 130 & 130 & 130 & 130 & 130 \\
\hline \multirow{2}{*}{} & Accuracy & $0.6^{\circ}$ & $0.6^{\circ}$ & $0.6^{\circ}$ & $0.6^{\circ}$ & $1.5^{\circ}$ & $3.5^{\circ}$ & $7.5^{\circ}$ & $15^{\circ}$ & $16^{\circ}$ & $20^{\circ}$ & $20^{\circ}$ \\
\cline { 2 - 11 } & $\operatorname{IMF}(\mathrm{dB})$ & 42 & 38 & 34 & 29 & 23 & 17 & 12 & 9 & 8 & 6.5 & 6.5 \\
\hline \hline
\end{tabular}

Table (3) The effect of the SIRi on MCMV and CTMV performances $(\mathrm{SNRi}=20 \mathrm{~dB})$

\begin{tabular}{|l|l|l|l|l|l|l|l|l|l|l|l|l|}
\hline \hline \multicolumn{2}{|l|}{ SIRi(dB) } & -25 & -20 & -15 & -10 & -5 & 0 & 5 & 10 & 15 & 20 & 25 \\
\hline \hline \multirow{2}{*}{} & Accuracy & $0.6^{\circ}$ & $0.6^{\circ}$ & $0.6^{\circ}$ & $0.6^{\circ}$ & $0.6^{\circ}$ & $0.6^{\circ}$ & $0.6^{\circ}$ & $0.6^{\circ}$ & $0.6^{\circ}$ & $0.6^{\circ}$ & $0.6^{\circ}$ \\
\cline { 2 - 12 } & $\operatorname{IMF}(\mathrm{dB})$ & 115 & 115 & 115 & 115 & 115 & 115 & 115 & 115 & 115 & 115 & 115 \\
\hline \multirow{2}{Z}{} & Accuracy & $0.6^{\circ}$ & $0.6^{\circ}$ & $0.6^{\circ}$ & $0.6^{\circ}$ & $0.6^{\circ}$ & $0.6^{\circ}$ & $1.6^{\circ}$ & $2.5^{\circ}$ & $9.5^{\circ}$ & - & - \\
\cline { 2 - 12 } & $\operatorname{IMF}(\mathrm{dB})$ & 51 & 47 & 44 & 40 & 36 & 32 & 26 & 19 & 10.5 & - & - \\
\hline \hline
\end{tabular}

Table (4) Effect of number of elements on MCMV and CTMV performances $(\mathrm{SNRi}=-5 \mathrm{~dB})$

\begin{tabular}{|l|l||l|l|l|l|l|l|l|}
\hline \hline \multicolumn{2}{|l|}{$\mathrm{M}$} & 3 & 5 & 10 & 15 & 20 & 25 & 30 \\
\hline \hline \multirow{2}{*}{$\sum_{\sum}$} & Accuracy & $0.6^{\circ}$ & $0.6^{\circ}$ & $0.6^{\circ}$ & $0.6^{\circ}$ & $0.6^{\circ}$ & $0.6^{\circ}$ & $0.6^{\circ}$ \\
\cline { 2 - 9 } & $\mathrm{IMF}(\mathrm{dB})$ & 130 & 130 & 130 & 130 & 130 & 130 & 130 \\
\hline \hline \multirow{2}{*}{} & Accuracy & $28^{\circ}$ & $25^{\circ}$ & $20^{\circ}$ & $10.5^{\circ}$ & $3.5^{\circ}$ & $1.5^{\circ}$ & $0.6^{\circ}$ \\
\cline { 2 - 9 } & $\mathrm{IMF}(\mathrm{dB})$ & 6 & 6.5 & 7 & 11 & 17 & 24 & 28 \\
\hline \hline
\end{tabular}

Table (5) Effect of number of elements on MCMV and CTMV performances $(\mathrm{SNRi}=20 \mathrm{~dB})$

\begin{tabular}{|c||l||l|l|l|l|l|l|l|}
\hline \hline \multicolumn{2}{|l|}{$\mathrm{M}$} & 3 & 5 & 10 & 15 & 20 & 25 & 30 \\
\hline \multirow{2}{*}{$\sum_{\sum}^{U}$} & Accuracy & $0.6^{\circ}$ & $0.6^{\circ}$ & $0.6^{\circ}$ & $0.6^{\circ}$ & $0.6^{\circ}$ & $0.6^{\circ}$ & $0.6^{\circ}$ \\
\cline { 2 - 9 } & $\mathrm{IMF}(\mathrm{dB})$ & 119 & 118 & 116 & 115 & 115 & 110 & 105 \\
\hline \hline$\sum$ & Accuracy & $20^{\circ}$ & $10^{\circ}$ & $4^{\circ}$ & $1.75^{\circ}$ & $0.6^{\circ}$ & $0.6^{\circ}$ & $0.6^{\circ}$ \\
\cline { 2 - 9 } & $\mathrm{IMF}(\mathrm{dB})$ & 6 & 10 & 18 & 25 & 32 & 35 & 42 \\
\hline \hline
\end{tabular}


Table (6) Effect of number snapshots on MCMV and CTMV performances $(\mathrm{SNRi}=-5 \mathrm{~dB})$

\begin{tabular}{|c|c|c|c|c|c|c|c|c|c|c|}
\hline \multicolumn{2}{|l|}{$\mathrm{N}$} & 2 & 4 & 8 & 16 & 32 & 64 & 128 & 256 & \\
\hline \multirow{2}{*}{$\sum_{\sum}^{Z}$} & Accuracy & $0.6^{\circ}$ & $0.6^{\circ}$ & $0.6^{\circ}$ & $0.6^{\circ}$ & $0.6^{\circ}$ & $0.6^{\circ}$ & $0.6^{\circ}$ & $0.6^{\circ}$ & $0.6^{\circ}$ \\
\hline & $\operatorname{IMF}(\mathrm{dB})$ & 130 & 130 & 130 & 130 & 130 & 130 & 130 & 130 & 130 \\
\hline \multirow{2}{*}{$\sum_{\in}^{B}$} & Accuracy & - & - & - & - & $9.5^{\circ}$ & $6.5^{\circ}$ & $4.5^{\circ}$ & $3.5^{\circ}$ & $3.5^{\circ}$ \\
\hline & $\operatorname{IMF}(\mathrm{dB})$ & - & - & - & - & 11 & 14 & 15.5 & 17 & 17 \\
\hline
\end{tabular}

Table (7) Effect of number snapshots on MCMV and CTMV performances $(\mathrm{SNRi}=20 \mathrm{~dB})$

\begin{tabular}{|c|c|c|c|c|c|c|c|c|c|c|}
\hline \multicolumn{2}{|l|}{$\mathrm{N}$} & 2 & 4 & 8 & 16 & 32 & 64 & 128 & 256 & 512 \\
\hline \multirow{2}{*}{$\sum_{\sum}^{Z}$} & Accuracy & $0.6^{\circ}$ & $0.6^{\circ}$ & $0.6^{\circ}$ & $0.6^{\circ}$ & $0.6^{\circ}$ & $0.6^{\circ}$ & $0.6^{\circ}$ & $0.6^{\circ}$ & $0.6^{\circ}$ \\
\hline & $\mathrm{IMF}(\mathrm{dB})$ & 122 & 122 & 122 & 121 & 120 & 120 & 118 & 117 & 115 \\
\hline \multirow{2}{*}{$\sum_{\mathcal{U}}^{Z}$} & Accuracy & - & - & - & - & $1^{\circ}$ & $0.6^{\circ}$ & $0.6^{\circ}$ & $0.6^{\circ}$ & $0.6^{\circ}$ \\
\hline & $\operatorname{IMF}(\mathrm{dB})$ & - & - & - & - & 28 & 30 & 32 & 32 & 32 \\
\hline
\end{tabular}

Table (8) Effect of DOA errors on MCMV and CTMV performances $(\mathrm{SNRi}=-5 \mathrm{~dB})$

\begin{tabular}{|c|c|c|c|c|c|c|c|c|c|c|}
\hline \multicolumn{2}{|c|}{ DOA error } & $(0,0)$ & $(1,0)$ & $(2,0)$ & $(0,1)$ & $(1,1)$ & $(2,1)$ & $(0,2)$ & $(1,2)$ & $(2,2)$ \\
\hline \multirow{2}{*}{$\sum_{\sum}^{Z}$} & Accuracy & $0.6^{\circ}$ & $0.6^{\circ}$ & $0.6^{\circ}$ & $1.6^{\circ}$ & $1.6^{\circ}$ & $1.6^{\circ}$ & $2.6^{\circ}$ & $2.6^{\circ}$ & $2.6^{\circ}$ \\
\hline & $\mathrm{IMF}(\mathrm{dB})$ & 130 & 130 & 130 & 25 & 25 & 25 & 20 & 20 & 20 \\
\hline \multirow{2}{*}{$\sum_{E}$} & Accuracy & $3.5^{\circ}$ & $4.5^{\circ}$ & $4.6^{\circ}$ & $4.6^{\circ}$ & $5.5^{\circ}$ & $5.6^{\circ}$ & $5.7^{\circ}$ & $6.5^{\circ}$ & $6.7^{\circ}$ \\
\hline & $\mathrm{IMF}(\mathrm{dB})$ & 17 & 15.5 & 15.5 & 15.5 & 15 & 14.5 & 14 & 13.5 & 13 \\
\hline
\end{tabular}

Table (9) Effect of DOA errors on MCMV and CTMV performances $(\mathrm{SNRi}=20 \mathrm{~dB})$

\begin{tabular}{|c|c|c|c|c|c|c|c|c|c|c|}
\hline \multicolumn{2}{|c|}{ DOA error } & $(0,0)$ & $(1,0)$ & $(2,0)$ & $(0,1)$ & $(1,1)$ & $(2,1)$ & $(0,2)$ & $(1,2)$ & $(2,2)$ \\
\hline \multirow{2}{*}{$\sum_{\sum}^{Z}$} & Accuracy & $0.6^{\circ}$ & $0.65^{\circ}$ & $0.65^{\circ}$ & $1.55^{\circ}$ & $1.6^{\circ}$ & $1.65^{\circ}$ & $2.6^{\circ}$ & $2.6^{\circ}$ & $2.75^{\circ}$ \\
\hline & $\operatorname{IMF}(\mathrm{dB})$ & 115 & 105 & 100 & 11 & 9 & 8 & 5 & 4.5 & 4 \\
\hline \multirow{2}{*}{$\sum_{\in}$} & Accuracy & $0.6^{\circ}$ & $0.75^{\circ}$ & $1.75^{\circ}$ & $0.75^{\circ}$ & $1.25^{\circ}$ & $2.75^{\circ}$ & $3^{\circ}$ & $3.25^{\circ}$ & $3.75^{\circ}$ \\
\hline & $\mathrm{IMF}(\mathrm{dB})$ & 32 & 30 & 27 & 29 & 22.5 & 22 & 21 & 20 & 18 \\
\hline
\end{tabular}




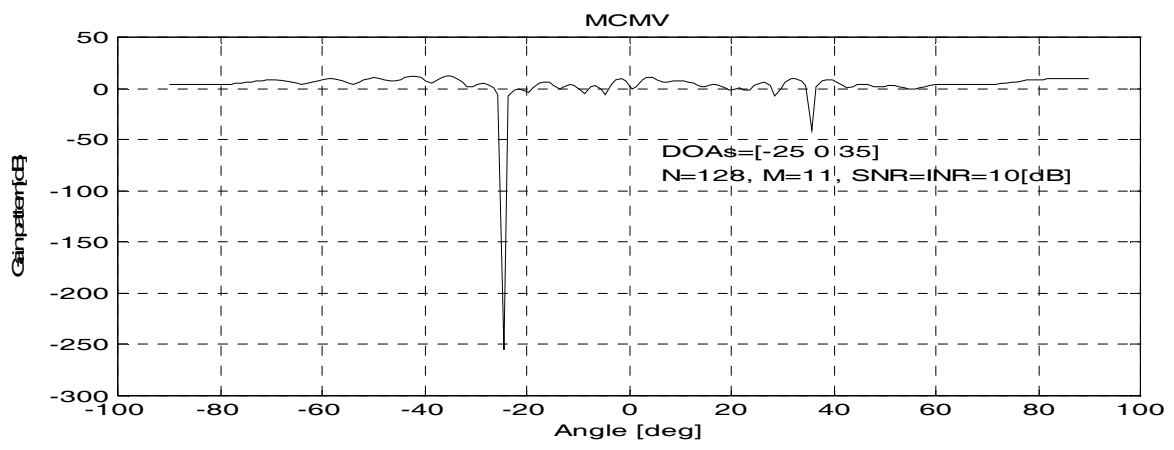

Fig.(1) Gain pattern of MCMV beamformer (one coherent, one uncorrelated interference)

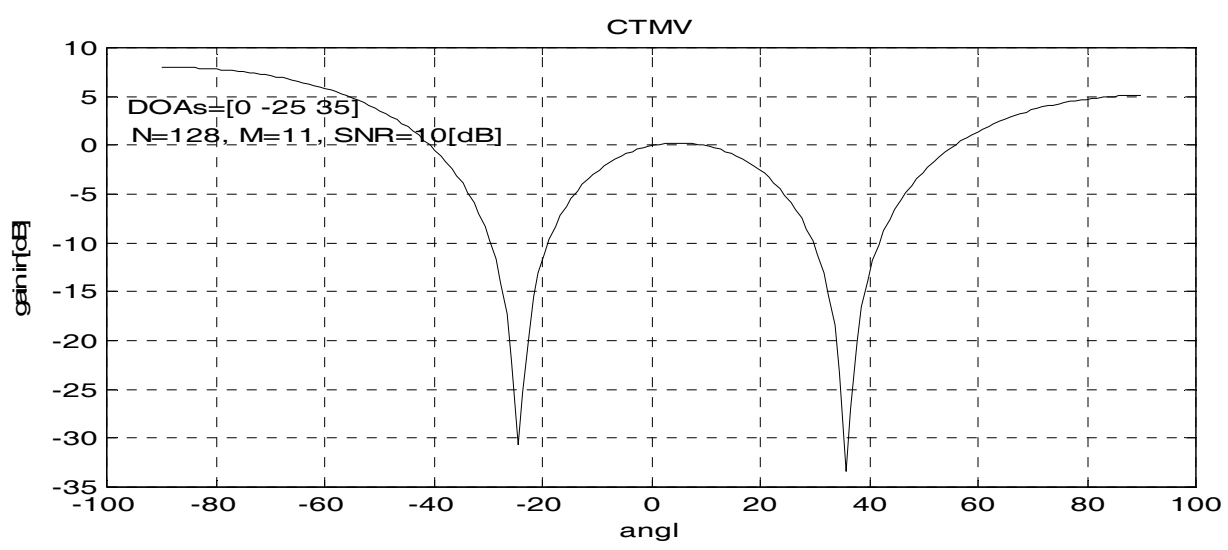

Fig.(2) Gain pattern of the CTMV beamformer (wideband signal and interference)

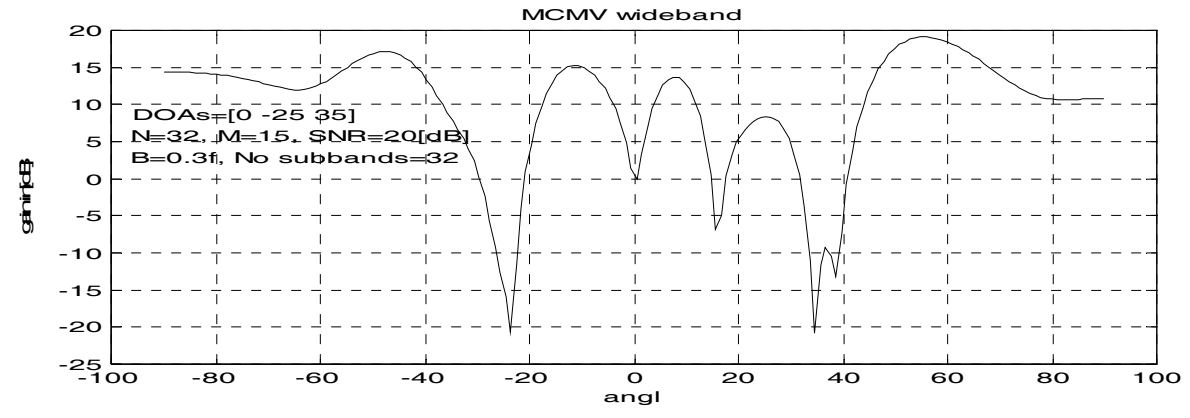

Fig.(3) Gain pattern of the MCMV beamformer (wideband signal and interference) 


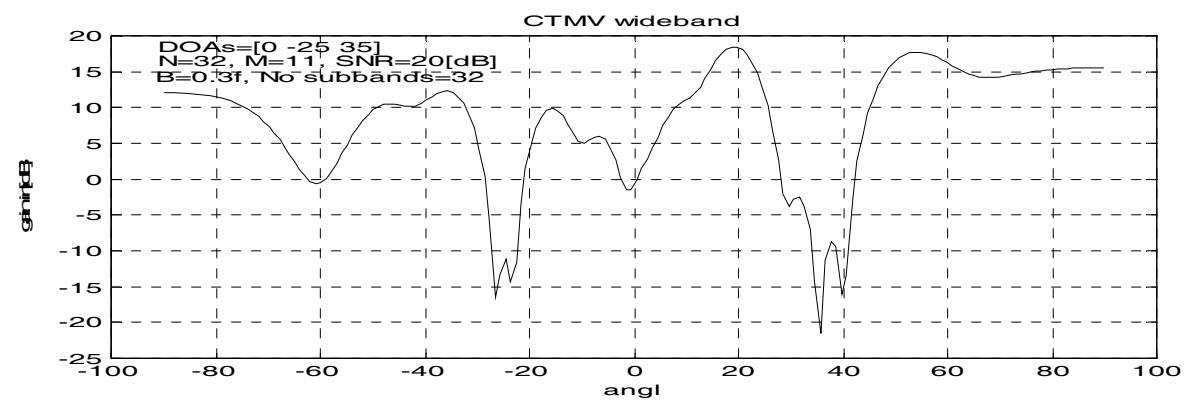

Fig.(4) Gain pattern of the CTMV beamformer (wideband signal and interference)

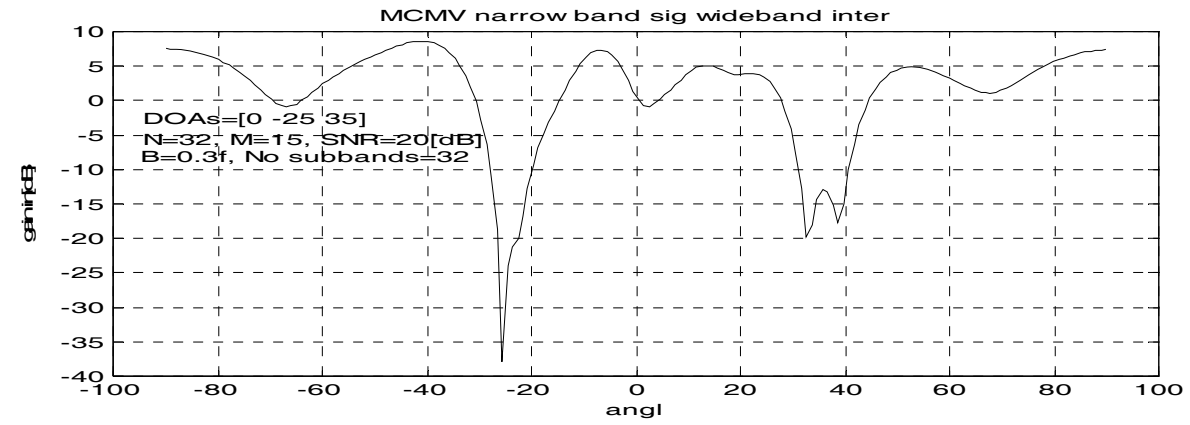

Fig(5) Gain pattern of MCMVbeamformer (narrowband signal \& wideband interference)

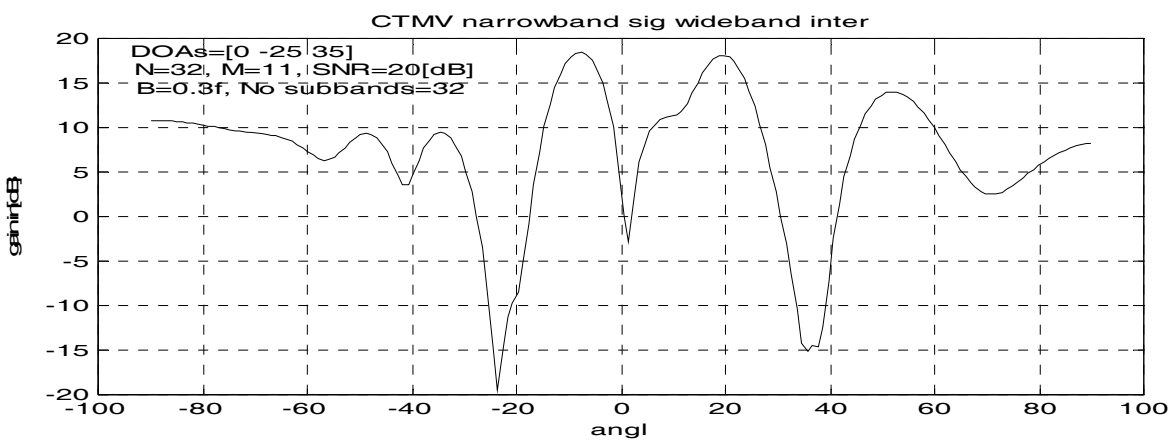

Fig.(6)Gain pattern of CTMV beamformer (narrowband signal \& wideband interfrence) 